\title{
Calculation of nucleon strange quark content with dynamical overlap quarks
}

\author{
JLQCD collaboration: K. Takeda ${ }^{* a}$, S. Aoki ${ }^{a, b}$, S. Hashimoto ${ }^{c, d}$, T. Kaneko ${ }^{c, d}$, \\ T. Onogi ${ }^{e}, \mathbf{N}$. Yamada ${ }^{c, d}$ \\ ${ }^{a}$ Graduate School of Pure and Applied Sciences, University of Tsukuba, Tsukuba, Ibaraki, \\ 305-8571, Japan \\ ${ }^{b}$ Riken BNL Research Center, Brookhaven National Laboratory, Upton, New York 11973, USA \\ ${ }^{c}$ High Energy Accelerator Research Organization (KEK), Ibaraki 305-0801, Japan \\ ${ }^{d}$ School of High Energy Accelerator Science, The Graduate University for Advanced Studies \\ (Sokendai), Ibaraki 305-0801, Japan \\ ${ }^{e}$ Department of Physics, Osaka University Toyonaka, Osaka 560-0043, Japan \\ E-mail: ktakeda@het.ph.tsukuba.ac.jp
}

\begin{abstract}
We calculate the nucleon strange quark content directly from disconnected three-point functions. Numerical simulations are carried out in two-flavor QCD using the overlap quark action with up and down quark masses down to a fifth of the physical strange quark mass. To improve the statistical accuracy, we calculate the nucleon two-point functions with the low-mode averaging technique, whereas the all-to-all quark propagator is used for the disconnected quark loop. We obtain the $y$ parameter, which is the ratio of the strange and light quark contents, $y=0.024(45)$ at the physical point. This is in a good agreement with our earlier calculation from the nucleon spectrum through the Feynman-Hellmann theorem.
\end{abstract}

The XXVII International Symposium on Lattice Field Theory - LAT2009

July 26-31 2009

Peking University, Beijing, China

${ }^{*}$ Speaker. 


\section{Introduction}

The nucleon strange quark content $\langle N|\bar{s} s| N\rangle$ is an important parameter to determine the cross section of the scattering of dark matter candidates from the nucleon [1], 2]]. It can not be measured directly by experiments, and only lattice QCD can provide a model-independent and nonperturbative determination. A precise lattice calculation is, however, very challenging, because only disconnected diagrams contribute to the strange quark content and they are computationally very expensive to calculate with the conventional method. In addition, the scalar operator $\bar{s} s$ has a vacuum expectation value (VEV), which diverges towards the continuum limit. We need to subtract the VEV contribution and this induces a substantial uncertainty in the strange quark content.

In our previous study [3], we avoid the above mentioned difficulties by calculating the strange quark content from the quark mass dependence of the nucleon mass $m_{N}$ through the FeynmanHellmann theorem

$$
\langle N|\bar{s} s| N\rangle=\frac{\partial m_{N}}{\partial m_{s}} .
$$

We refer to this method as the spectrum method in the following. This method is, however, not applicable to other interesting matrix elements, such as the strange quark spin fraction of the nucleon. In this article, therefore, we attempt a direct determination of strange quark content from nucleon matrix element including a disconnected diagram. To this end, we employ the overlap quark action, which has exact chiral symmetry, and improved measurement methods, such as the low-mode averaging (LMA) technique [4, 5] and the use of the all-to-all quark propagator [6].

\section{Simulation details}

Gauge ensembles of two-flavor QCD are generated on a $L^{3} \times T=16^{3} \times 32$ lattice using the Iwasaki gauge action and the overlap quark action. We set the gauge coupling $\beta=2.30$ at which the lattice spacing determined from the Sommer scale $r_{0}=0.49 \mathrm{fm}$ is $a=0.118(2) \mathrm{fm}$. Our simulation is accelerated by introducing a topology fixing term into our lattice action [7], and we simulate only the trivial topological sector $Q=0$ in this study. We take four values of bare up and down quark masses $m_{u d}=0.015,0.025,0.035$ and 0.050 , which cover a range of the pion mass $m_{\pi}=$ $290-520 \mathrm{MeV}$. Statistics are 100 independent configurations at each quark mass. We refer readers to [8] for further details on our configuration generation. In our measurement, we take two values of the valence strange quark mass $m_{s, v a l}=0.070$ and 0.100 , which are close to the physical mass $m_{s, p h y s}=0.077$ determined from our analysis of the meson spectrum 9 ].

The strange quark content can be extracted from nucleon two- and three-point functions

$$
\begin{aligned}
C_{2 p t}^{\Gamma}(t, \Delta t) & =\operatorname{Tr}[\Gamma\langle N(t+\Delta t) \bar{N}(t)\rangle] \\
C_{3 p t}^{\Gamma}\left(t, \Delta t, \Delta t_{s}\right) & =\operatorname{Tr}\left[\Gamma\left\langle N(t+\Delta t) S\left(t+\Delta t_{s}\right) \bar{N}(t)\right\rangle\right]-\left\langle S\left(t+\Delta t_{s}\right)\right\rangle \operatorname{Tr}[\Gamma\langle N(t+\Delta t) \bar{N}(t)\rangle],
\end{aligned}
$$

where $S=\bar{s} s$ is the strange scalar operator, $t$ represents the temporal coordinate of the nucleon source operator, and $\Delta t\left(\Delta t_{s}\right)$ is the temporal separation between the nucleon source and sink (quark loop). We calculate $C_{2 p t}^{\Gamma}$ and $C_{3 p t}^{\Gamma}$ with two choices of the projector $\Gamma=\Gamma_{ \pm}=\left(1 \pm \gamma_{4}\right) / 2$ corresponding to the forward and backward propagation of the nucleon. We then take the average over 
the two choices of $\Gamma$ with appropriately chosen temporal separations $\Delta t$ and $\Delta t_{s}$. The averaged correlators, which are denoted by $C_{2 p t}$ and $C_{3 p t}$ in the following, show reduced statistical fluctuation.

For further improvement of the statistical accuracy, we employ the low-mode averaging(LMA) technique [4, 5] to calculate $C_{2 p t}$ and $C_{3 p t}$. In this method, the quark propagator is expanded in terms of the eigenmodes of the Dirac operator $D$. We calculate the contribution of 100 low-modes exactly as

$$
(D)_{\text {low }}^{-1}=\sum_{i=1}^{100} \frac{1}{\lambda^{(i)}} v^{(i)} v^{(i) \dagger}, \quad D v^{(i)}=\lambda^{(i)} v^{(i)} .
$$

The remaining contribution from the higher modes is taken from by that of the conventional pointto-all propagator. With this decomposition of the quark propagator, $C_{2 p t}$ is divided into eight contributions

$$
C_{2 p t}=C_{2 p t}^{L L L}+C_{2 p t}^{L L H}+C_{2 p t}^{L H L}+C_{2 p t}^{H L L}+C_{2 p t}^{L H H}+C_{2 p t}^{H L H}+C_{2 p t}^{H H L}+C_{2 p t}^{H H H} .
$$

It is expected that $C_{2 p t}^{L L L}$ dominates $C_{2 p t}$ at large temporal separation $\Delta t$. The statistical accuracy of $C_{2 p t}$ can be remarkably improved by averaging $C_{2 p t}^{L L L}$ over the location of the nucleon source operator. We also improve the statistical accuracy of other contributions $\left(C_{2 p t}^{L L H}, \ldots, C_{2 p t}^{H H H}\right)$ by using point-to-all propagators averaged over 4 or 8 different source locations. The nucleon piece of the disconnected correlator $C_{3 p t}$ is calculated in the same way.

Since the nucleon correlators $C_{2 p t}$ and $C_{3 p t}$ damp rapidly as $\Delta t$ increases, it is essential to reduce the contamination from excited states at small $\Delta t$. In this study, we employ the Gaussian smearing

$$
q_{s m r}(\mathbf{x}, t)=\sum_{\mathbf{y}}\left\{\left(1+\frac{\omega}{4 N} H\right)^{N}\right\}_{\mathbf{x}, \mathbf{y}} q(\mathbf{y}, t), \quad H_{\mathbf{x}, \mathbf{y}}=\sum_{i=1}^{3}\left(\delta_{\mathbf{x}, \mathbf{y}-i}+\delta_{\mathbf{x}, \mathbf{y}+i}\right)
$$

for both of the source and sink operators. The parameters $\omega=20$ and $N=400$ are chosen so that the effective mass of $C_{2 p t}$ shows a good plateau. For comparison, we repeat our measurement with the local sink operator. In this additional measurement, we test the local and an exponential source operator $q_{s m r}(\mathbf{x}, t)=\sum_{\mathbf{r}} \exp (-B|\mathbf{r}|) q(\mathbf{x}+\mathbf{r}, t)$. The parameter $B$ is chosen so that the distribution of the smeared quark is close to that of the Gaussian smearing (2.5).

To calculate the disconnected quark loop in $C_{3 p t}$, we construct the all-to-all quark propagator as proposed in 6 . The low-mode contribution is the same as in (2.3) and the contribution from the high-modes is estimated by employing the noise method with the dilution technique [6]. We prepare a single $Z_{2}$ noise vector $\eta$ for each configuration and it is split into $N_{d}=3 \times 4 \times T / 2$ vectors $\eta^{(d)}\left(d=1, \ldots, N_{d}\right)$ which have non-zero elements only for single color and spinor indices and two consecutive time-slices. The high-mode contribution is then given by

$$
(D)_{h i g h}^{-1}=\sum_{d=1}^{N_{d}} \psi^{(d)} \eta^{(d) \dagger}
$$

where $\psi^{(d)}$ is the solution of the linear equation

$$
D \psi^{(d)}=\left(1-\mathscr{P}_{\text {low }}\right) \eta^{(d)}
$$

and $\mathscr{P}_{\text {low }}$ is the projection operator to the subspace spanned by the low-modes. 


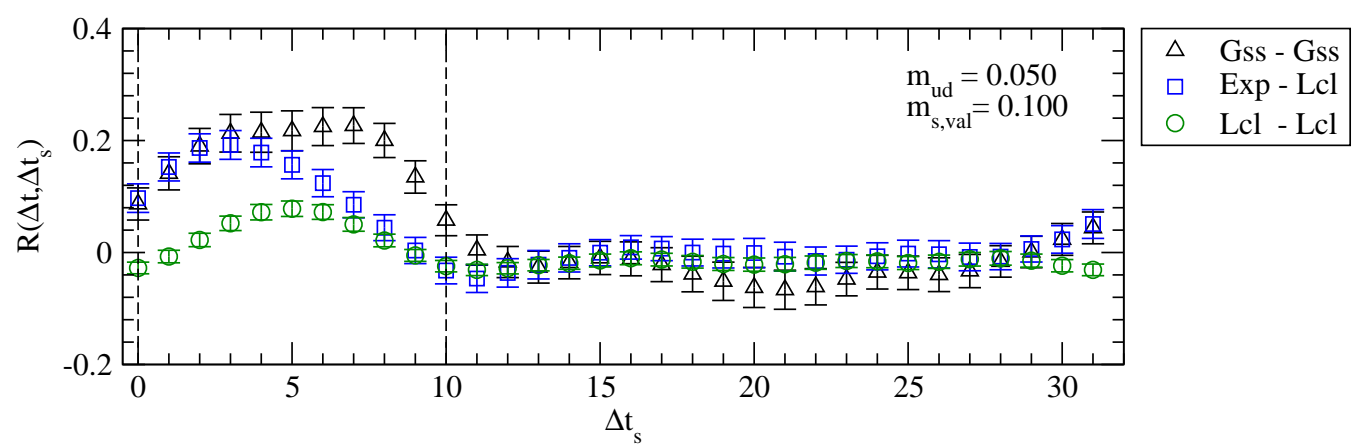

Figure 1: Ratio $R\left(\Delta t, \Delta t_{s}\right)$ at $m_{u d}=0.050$ and $m_{s, v a l}=0.100$ with $\Delta t$ fixed to 10 . Triangles show data with Gaussian smeared source and sink, whereas circles (squares) are with the local sink and local (exponentially smeared) source. We omit the noisy high-mode contribution to the quark loop in this plot. Vertical lines show the location of the source and sink operators.

We also tested the all-to-all quark propagator to calculate the high-mode contributions $C_{2 p t}^{L L H}, \ldots, C_{2 p t}^{H H H}$ in (2.4). It turned out, however, that these contributions have large statistical error due to the insufficient number of the noise samples. We therefore use $C_{2 p t}$ and $C_{3 p t}$ calculated with the LMA in the following analysis.

\section{Matrix element at simulated quark masses}

We extract the unrenormalized matrix element $\langle N|\bar{s} s| N\rangle_{\text {lat }}$ from the ratio

$$
R\left(\Delta t, \Delta t_{s}\right)=\frac{C_{3 p t}\left(t, \Delta t, \Delta t_{s}\right)}{C_{2 p t}(t, \Delta t)} \underset{\Delta t, \Delta t_{s} \rightarrow \infty}{\longrightarrow}\langle N|\bar{s} s| N\rangle_{l a t} .
$$

Figure 1 shows $\Delta t_{s}$ dependence of $R\left(\Delta t, \Delta t_{s}\right)$ at our heaviest $u d$ quark mass $m_{u d}=0.050$ with a fixed $\Delta t$. We observe a clear plateau between the nucleon source and sink with the Gaussian smeared operator. On the other hand, the plateau is unclear if the local operator is used for the source and/or sink. It is therefore crucial for a reliable determination of $\langle N|\bar{s} s| N\rangle_{\text {lat }}$ from $R\left(\Delta t, \Delta t_{s}\right)$ to reduce contamination from the excited states by appropriately smearing the nucleon operators.

The situation is similar at two smaller $u d$ quark masses $m_{u d}=0.035$ and 0.025 . As shown in Fig. 2, however, we do not observe a clear signal even with the smeared source and sink at our smallest quark mass $m_{u d}=0.015$. To observe a clear plateau of $R\left(\Delta t, \Delta t_{s}\right)$ at such small $m_{u d}$, we

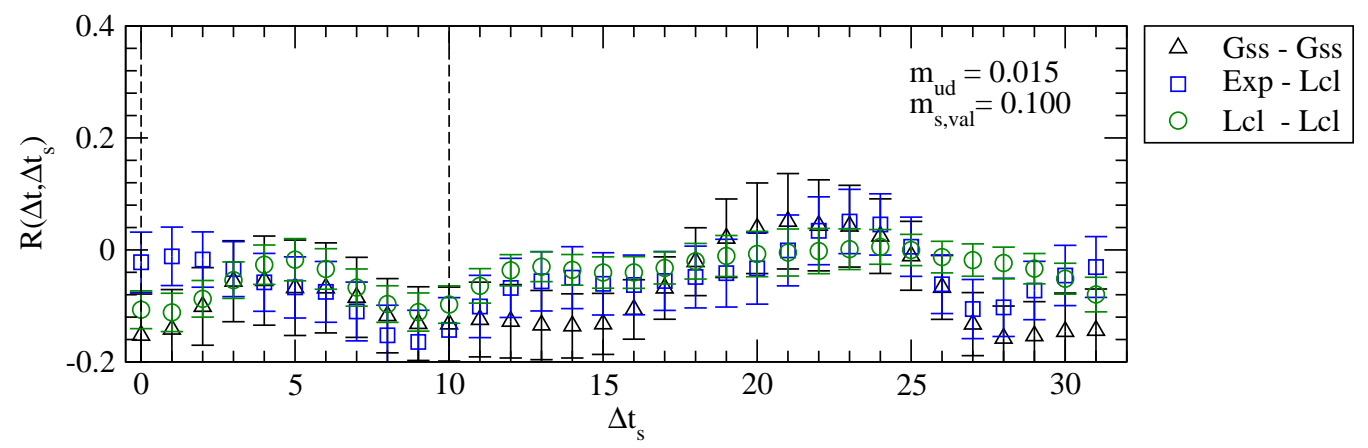

Figure 2: Ratio $R\left(\Delta t, \Delta t_{s}\right)$ at $m_{u d}=0.015$ and $m_{s, v a l}=0.100$. 

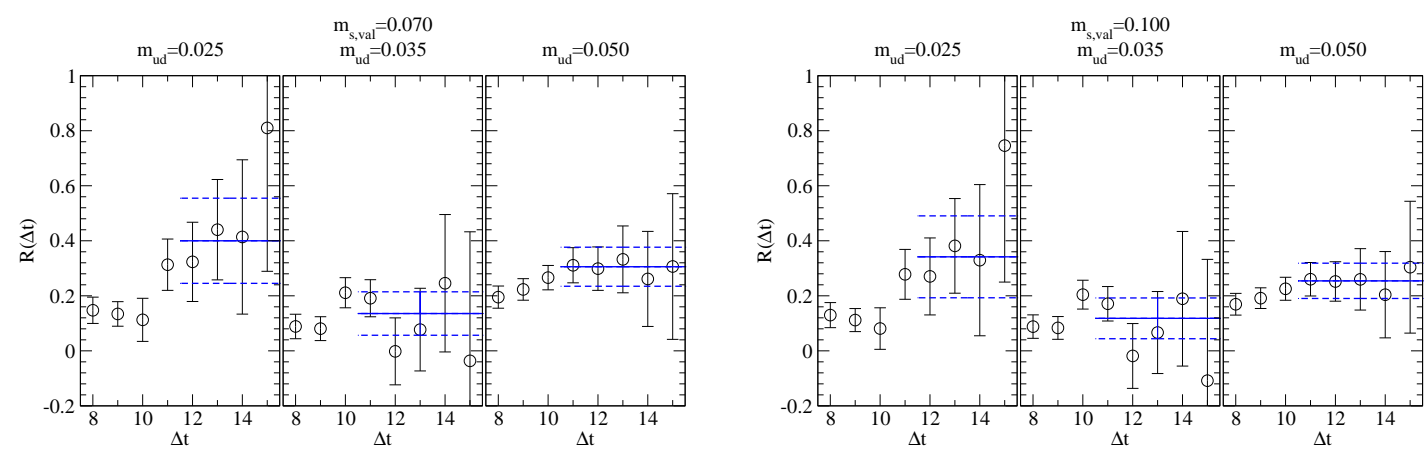

Figure 3: Ratio $R(\Delta t)$ as a function of $\Delta t$. Three left (right) panels show data at $m_{s, v a l}=0.070(0.100)$. The horizontal lines show the constant fit to $R(\Delta t)$ in terms of $\Delta t$ to determine $\langle N|\bar{s} s| N\rangle_{\text {lat }}$.

may need more statistics as well as a larger lattice to suppress finite volume corrections, which are possibly sizable at $M_{\pi} L \sim 2.8$ at $m_{u d}=0.015$. We leave such a calculation for future studies, and omit data at this $m_{u d}$ in the following analysis.

In this report, $\langle N|\bar{s} s| N\rangle_{\text {lat }}$ is determined by the following simple two-step fits. First, we carry out a constant fit to $R\left(\Delta t, \Delta t_{s}\right)$ in terms of $\Delta t_{s}$. The fit result, which we denote by $R(\Delta t)$, is plotted as a function of $\Delta t$ in Fig. B. We then extract $\langle N|\bar{s} s| N\rangle_{\text {lat }}$ by a constant fit to $R(\Delta t)$ at $12 \leq \Delta t \leq 15$. As seen in Fig. 通, $R(\Delta t)$ do not show significant $\Delta t$ dependence with this range of $\Delta t$. We therefore expect that extraction of ground state signal is well under control.

\section{Strange quark content at physical point}

As seen in Fig. 3, the fit result for $\langle N|\bar{s} s| N\rangle_{\text {lat }}$ does not have significant $m_{s, v a l}$ dependence at each $m_{u d}$. This leads us to interpolate $\langle N|\bar{s} s| N\rangle_{\text {lat }}$ to the physical strange quark mass $m_{s, p h y s}=0.077$ using a linear form in terms of $m_{s, v a l}$. Fit results as well as fitted data at $m_{s, v a l}$ are plotted as a function of $m_{u d}$ in Fig. 4 .

At next-to-leading order of heavy baryon chiral perturbation theory (HBChPT) [10] the nucleon mass $m_{N}$ can be written as $m_{N}=m_{0}+C_{1} m_{u d}+C_{2} m_{s}+C_{3} m_{\pi}^{3}+C_{4} m_{K}^{3}+C_{5} m_{\eta}^{3}$, where $C_{i}$ $(i=1, \ldots, 5)$ are functions of the low-energy constants (LECs) in HBChPT. We note that the contributions from decuplet baryons are neglected. The Feynman-Hellmann theorem (11.1) then implies that the $m_{s}$ dependence of $\langle N|\bar{s} s| N\rangle$ comes from the $O\left(m_{K, \eta}^{3}\right)$ terms from $K$ and $\eta$ loops. By using the leading order relation $m_{K}^{2} \propto m_{u d}+m_{s}$ and $m_{\eta}^{2} \propto m_{u d}+2 m_{s}$, we obtain

$$
\langle N|\bar{s} s| N\rangle=\frac{\partial m_{N}}{\partial m_{s}}=D_{0}+D_{1} m_{u d}+O\left(m_{u d}^{2}\right)
$$

where $D_{0}$ and $D_{1}$ depend on the LECs and $m_{s}$.

We extrapolate $\langle N|\bar{s} s| N\rangle_{\text {lat }}$ at $m_{s, p h y s}$ by the linear form (4.1) with $D_{0,1}$ treated as fitting parameters. This chiral extrapolation is plotted in Fig. 4. We obtain $\langle N|\bar{s} s| N\rangle=0.11(21)$ at the physical point, where the error is statistical only. This is converted to the phenomenologically relevant parameters

$$
f_{T_{s}} \equiv \frac{m_{s, p h y s}\langle N|\bar{s} s| N\rangle}{m_{N}}=0.015(28)
$$




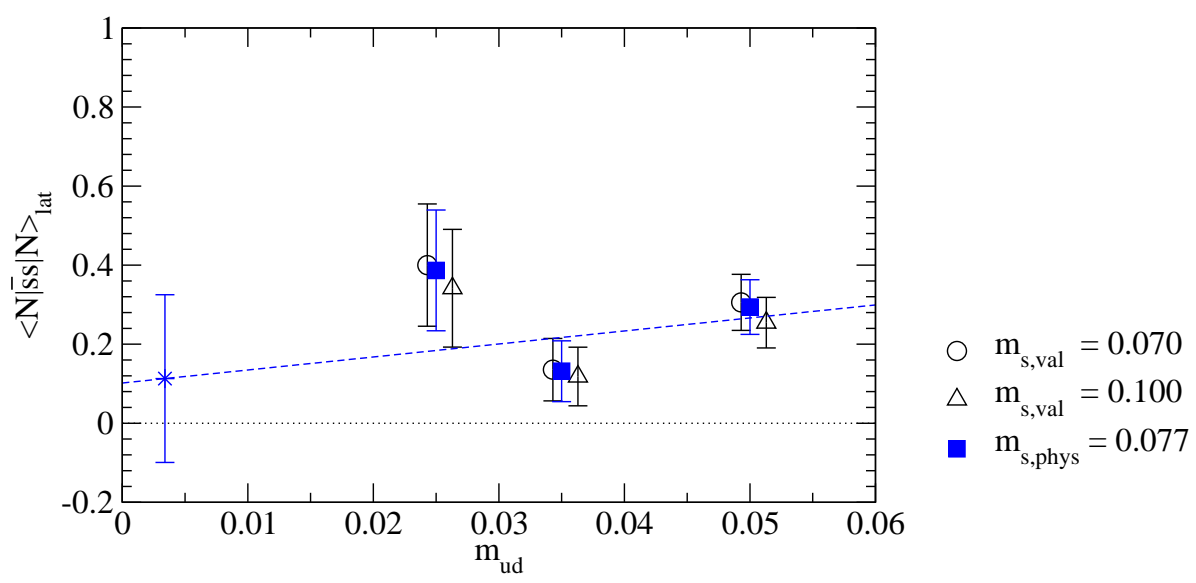

Figure 4: Chiral extrapolation of $\langle N|\bar{s}| N\rangle_{l a t}$ at $m_{s, p h y s}$ as a function of $m_{u d}$. For a comparison, we also plot $\langle N|\bar{s} s| N\rangle_{\text {lat }}$ at simulated strange quark masses $m_{s, v a l}=0.070$ and 0.100 .

and

$$
y \equiv \frac{2\langle N|\bar{s} s| N\rangle}{\langle N|\bar{u} u+\bar{d} d| N\rangle}=0.024(45)
$$

where we use the nucleon mass $m_{N}$ [11] and the $u d$ quark content $\langle N|\bar{u} u+\bar{d} d| N\rangle$ obtained in our previous study [3].

As shown in Fig. 5, we observe a good agreement with our estimate from the spectrum method [3]. The same figure also shows that previous studies with the Wilson-type fermions [12, 13, 14] led to rather large values for the strange quark content $y$. It is argued in [3, 15] that the explicit chiral symmetry breaking induces a mixing between the scalar operators of sea and valence quarks and leads to a substantial uncertainty in the strange quark content.

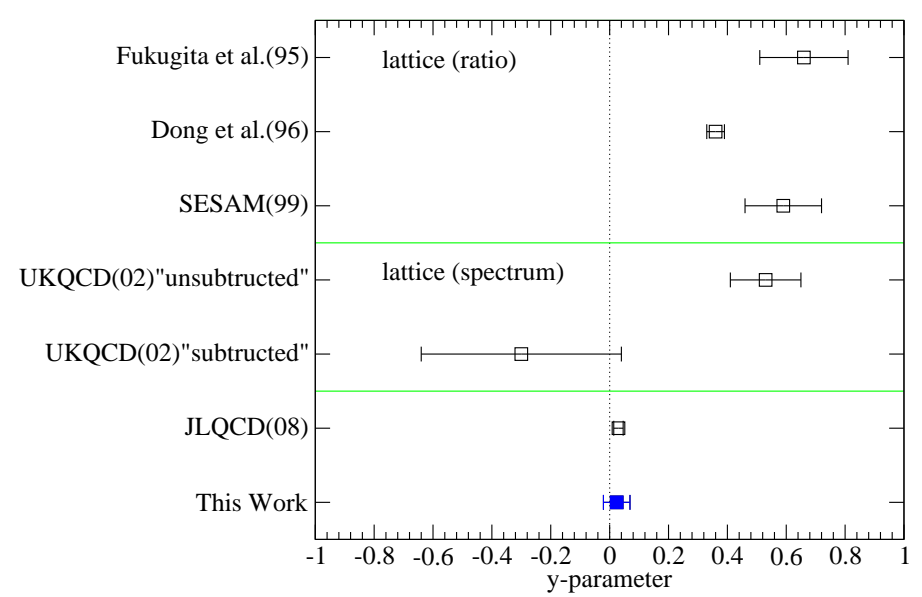

Figure 5: Comparison of $y$ parameter with previous estimates [3, 12, 13, 14, 15]. Our previous study [3] employs the overlap action, whereas other studies use the Wilson-type quark actions. Two results from the UKQCD study [15] are obtained with and without subtracting the unphysical effect due to the operator mixing. 


\section{Conclusion}

In this article, we report on our calculation of the strange quark content directly from the nucleon matrix element. We determine $f_{T_{s}}$ and $y$ with an accuracy of $O\left(10^{-2}\right)$. The key points leading to this accuracy are the use of the improved measurement techniques, namely the LMA and the all-to-all quark propagator, as well as the appropriately smeared operator both for nucleon source and sink. It is an interesting subject in the future to extend this study to other matrix elements containing disconnected diagram such as the quark spin fraction of the nucleon.

We observe a good agreement with our previous estimate from the spectrum method. Chiral symmetry preserved by the overlap action plays a crucial role in avoiding the unwanted operator mixing for the Wilson-type actions. For more precise determination, we need to extend our calculation to $N_{f}=2+1$ QCD and larger volumes. Our preliminary estimate with the spectrum method is reported at this conference [16]. A direct determination from nucleon disconnected functions in $N_{f}=2+1$ QCD is also in progress.

Numerical simulations are performed on Hitachi SR11000 and IBM System Blue Gene Solution at High Energy Accelerator Research Organization (KEK) under a support of its Large Scale Simulation Program (No. 09-05). This work is supported in part by the Grant-in-Aid of the Ministry of Education (No. 19540286, 20105001, 20105002, 20105003, 20340047, 21674002 and 21684013).

\section{References}

[1] E. A. Baltz, M. Battaglia, M.E. Peskin and T. Wizansky, Phys. Rev. D 74, 103521 (2006)

[2] J. Ellis, K.A. Olive, C. Savage, Phys. Rev. D 77, 065026 (2008) [0801 . 3656 [hep-ph ] ].

[3] H. Ohki et. al. (JLQCD collaboration), Phys. Rev. D 78, 054502 (2008) [0806 . 4744 [hep-lat ] ].

[4] L. Giusti, P. Hernandez, M. Laine, P. Weisz and H. Wittig, JHEP 0404, 013 (2004)

[5] T.A. DeGrand and S. Schaefer, Comput. Phys. Commun. 159, 185 (2004) [hep-lat/ 0401011 ].

[6] J. Foley et. al. (TrinLat collaboration), Phys. Commun 172, 145 (2005) [hep-lat/ 0505023 ].

[7] H. Fukaya et al. (JLQCD collaboration), Phys. Rev. D 74, 094505 (2006) [hep-lat / 0607020 ].

[8] S. Aoki et al. (JLQCD collaboration), Phys. Rev. D 78, 014508 (2008) [0803 . 3197 [hep-lat ] ].

[9] J. Noaki et. al. (JLQCD collaboration), in preparation.

[10] A. Walker-Loud, Nucl. Phys. A 747, 476 (2005) [hep-lat / 0405007 ].

[11] C. Amsler,et al. (Particle Data Group). Phys. lett. B 667, 1 (2008).

[12] M. Fukugita et al., Phys. Rev. D 51, 5319 (1995) [hep-lat/9408002].

[13] S.J. Dong, J.F. Lagae and K.F. Liu, Phys. Rev. D 54, 5496 (1996) [hep-ph / 9602259 ].

[14] S. Gusken et al. (SESAM collaboration), Phys. Rev. D 59, 054504 (1999) [hep-lat/9809066].

[15] C. Michael, C. McNeile and D. Hepburn (UKQCD collaboration), Nucl.Phys.Proc.Suppl. 106, 293 (2002) [hep-lat/0109028].

[16] H. Ohki et al. (JLQCD collaboration), in these proceedings. 